\title{
An integrated approach for validating micro mechanical systems based on simulation and test
}

\author{
Albert Albers · Tobias Deigendesch • \\ Hans-Georg Enkler · Stefan Hauser • \\ Pablo Leslabay $\cdot$ Jochen Oerding
}

Received: 26 October 2007/Accepted: 19 March 2008/Published online: 14 May 2008

(C) Springer-Verlag 2008

\begin{abstract}
Miniaturization of macroscopic mechanical systems enables the opening of new areas of application for micro technological systems. Because of technological restrictions, especially when applying miniaturized conventional manufacturing techniques, shape and material deviations cannot be scaled down in the same dimension like micro parts. Thus, the long-term objective is to ensure the functioning by appropriate design measures. In doing so, determination of the transfer behavior by modeling and simulation is required. This work presents two ways for ensuring the required properties of micro gears and describes how the results do correlate. The experimental way uses the radial composite inspection as it is used in the macroscopic world. The simulative way deploys a rolling simulation by utilization of finite element analysis. The virtual prototypes are originated on measured real test gears. When comparing experiment and simulation of the rolling behavior, in some short and long wave areas
\end{abstract}

\author{
A. Albers $(\square)$ - T. Deigendesch · H.-G. Enkler · S. Hauser . \\ P. Leslabay $\cdot$ J. Oerding \\ IPEK-Institute of Product Development, Universität Karlsruhe \\ (TH), Kaiserstraße 10, 76131 Karlsruhe, Germany \\ e-mail: albers@ipek.uni-karlsruhe.de \\ T. Deigendesch \\ e-mail: deigendesch@ipek.uni-karlsruhe.de \\ H.-G. Enkler \\ e-mail: enkler@ipek.uni-karlsruhe.de \\ S. Hauser \\ e-mail: hauser@ipek.uni-karlsruhe.de \\ P. Leslabay \\ e-mail: leslabay@ipek.uni-karlsruhe.de \\ J. Oerding \\ e-mail: oerding@ipek.uni-karlsruhe.de
}

deviations can be recognized. These can be ascribed to the reduction from three to two dimensions when modeling. Other deviations might be based on inaccuracies when mounting into the test rig. In other areas quite good correlations of test and simulation could be ascertained.

\section{Introduction and motivation}

Increasing miniaturization of mechanical systems implicates new challenges for the development, production and quality assurance. Due to technological restrictions, especially when apply miniaturized manufacturing techniques from the macro world shape and material deviations cannot be scaled down in the same way.

Respecting the influence of such deviations during the very early stages the product development process is of maximum importance (Marz 2005). For supporting the design process, new methods are required that enable the designer to model the later system behavior reliably.

The present work demonstrates two ways for ensuring the desired properties of micro gears and describes how the results correlate (cp. Fig. 1). As an experimental method the radial composite inspection is depicted, which is an accepted test for macro gears. The simulative method is a rolling simulation applying the finite element analysis. The virtual test gears are modelled on the basis of measured real gears and not on the nominal shape. The long term objective for an early assurance of system properties is to destermine the transfer behaviour and to derive adequate design measures. In doing so function fulfilment and thus a robust design can be provided.

The fundament for the determination of the validity of the model is a comparison with test of real micro gears. 


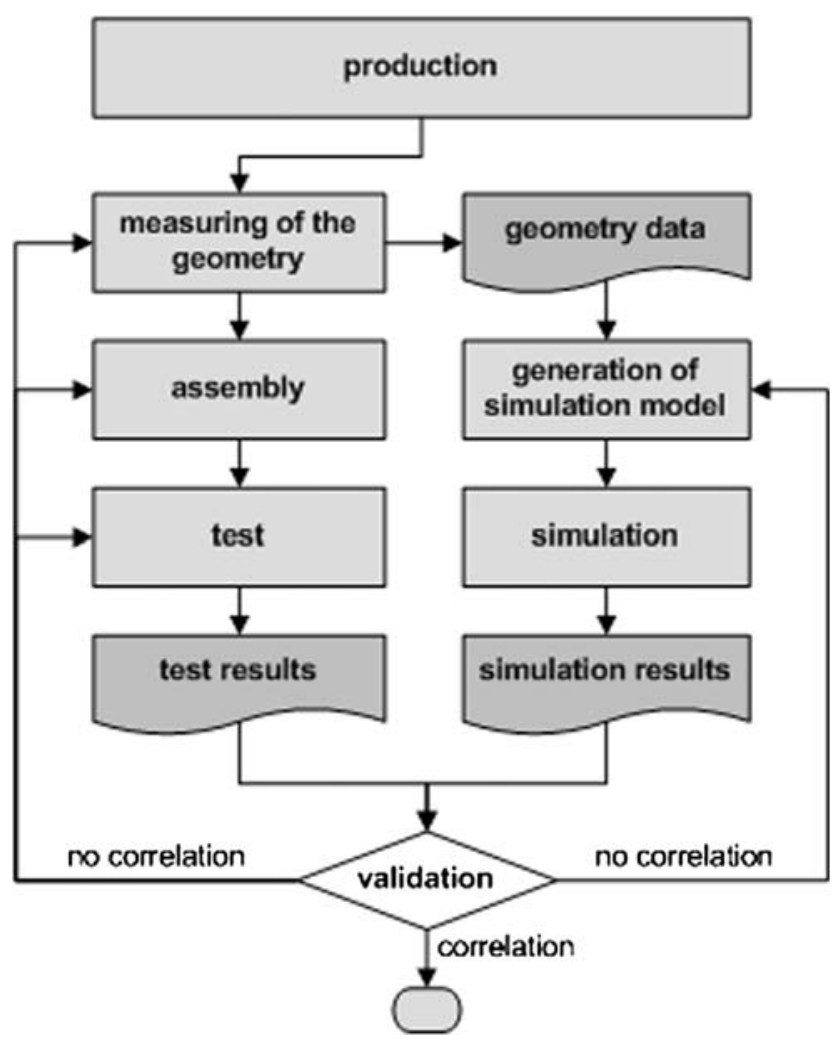

Fig. 1 Approach

Only in case of validity, the virtual model can be extrapolated to such designs that are impossible to test. Because of reaching the cutting edge of connection, joining and test technology for micro parts, measurement range and measurement deviation might be in the same dimension. Thus, a valid forecast of properties is a necessity for further product miniaturization.

For revision of this approach first tests were made with off-the-shelf plastic gears (module $1 \mathrm{~mm}$ ). The actual specimens a high stressable ceramic micro gears (cp. Fig. 2) made of zirconium oxide with a module of $169 \mu \mathrm{m}$ and a tooth width of $156 \mu \mathrm{m}$. which were developed, produced and measured within the Collaborative Research Center (CRC) 499 of the German Research Foundation.

Fig. 1 schematically depicts the approach.

\section{Measurement and modeling}

For simulation of the test an appropriate model has to be set up. First of all, the geometry of the particular component has to be created virtually.

In order to establish a basis for this modeling, the geometries of the gearwheels have been comprised by means of a tactile coordinate measuring machine (cp. Fig. 3) and special measuring software (Zeiss 2007;

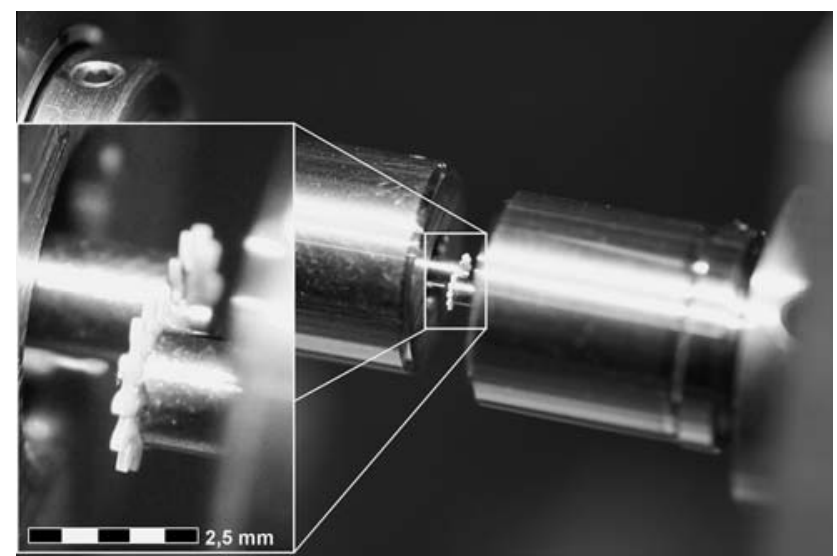

Fig. 2 CRC-gearwheels in micro test rig

Mitutoyo 2007). Though, application of commercial systems offers the advantage of being proven, tested and certified.

In principal, it is possible to generate a three dimensional model of the individual components. However, a simulation on the basis of three dimensional models increases computational effort considerably. In addition, components have to be measured in multiple layers. For this reason, a two dimensional approach was chosen for this work, i.e. all components have been reduced to two dimensions. Validity of this simplification has to be checked in the following.

To set up a model, measuring points of the profile in the midplane of the gearwheel were regarded.

Since not all measuring points lie exactly within one plane, they are projected onto one common plane initially. Afterwards the projected points are connected by means of a spline (Schoenberg 1969) that replicates the outline of the component. Simulation is based on the finite element method (FEM), i.e. the geometric model has to be discretized. Hence, the real component is being simulated by a

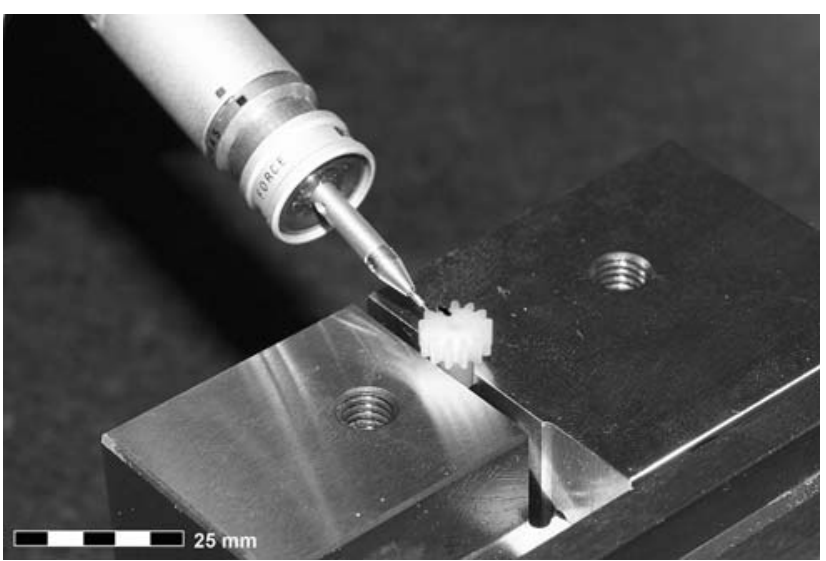

Fig. 3 Gearwheel measuring via tactile coordinate measuring machine 
finite number of data points as usual in measuring. Measuring points can be used directly to generate a finite element (FE) mesh. Thereto, measuring points should be equidistant, especially at the tooth flanks so that an adequate mesh for the subsequent analysis can be generated. For this work, the former approach was used, i.e. the mesh is based on a spline.

For generating virtual geometries an automated process has been set up generating a geometry that is prepared for the FE Preprocessor and based on the measuring points. The number of measuring points that are taken for geometry creation can be influenced by means of a reduction factor if desired. The number of points that are taken into account is geared to the element size of the subsequently generated FE mesh.

To analyze deviations of the real is-profile from the desired profile and to investigate the influence of these deviations on the system via the subsequently obtained simulation results and tests, a curvature analysis of the individual flanks can be used, for instance. Figure 4 shows the curvature, i.e. the turnaround per unit length, exemplarily for a measured and the desired tooth geometry (up to the base circle). In general, further options such as tipping angle of the individual teeth are cogitable.
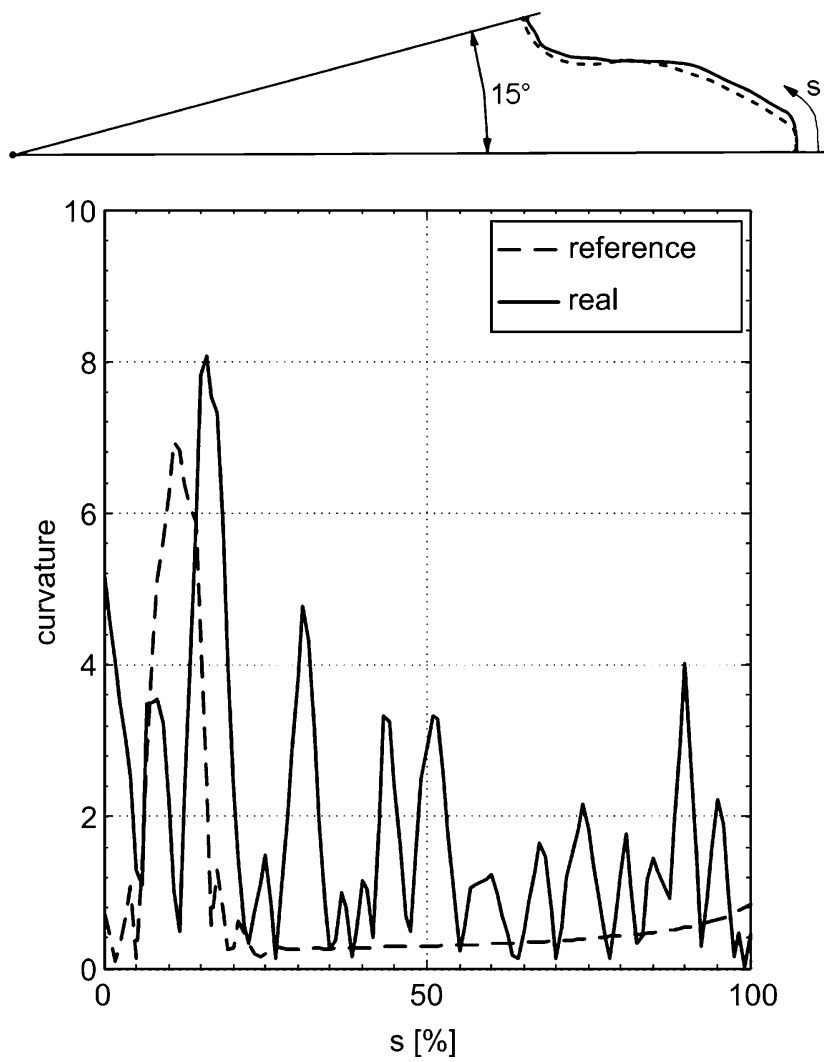

Fig. 4 Comparison of the curvature of a real tooth flank and the desired flank profile (up to base circle)

\section{Radial composite inspection}

The radial composite inspection is a test used in macro world for quality assurance of gearwheels (VDI 2001) in which a pair of gearwheels is being revolved free from clearance. This is achieved by a radial pre-load of the gearwheels in such a way as to constitute an engagement both on the left and the right flank (Fig. 5). Driving one of the gearwheels, the second one that is locomotive linearly dodges due to geometry deviations. Quality of gearwheels can then be evaluated and compared on the basis of the highest radial deviation difference.

In general, master gearwheels [NaFuO 1972a, b] that have to be at least two quality levels better than the tested gearwheels (NAN 1987) are used for such an evaluation. In this way, measured deviations can be related to the test gearwheel. But regarding molded micro mechanical systems such master gearwheels with higher quality level would have to be manufactured with an unjustifiable additional effort. In addition, currently there is a lack of accordant norms inclusive a definition of an applicable definition of the quality term.

For this work no master gearwheels but two test gearwheels have been coupled. The measured deviations in axis-center distances therefore cannot be related to one single gearwheel but the system of both. However, by systematic measurements of multiple gear tooth pairings of one and the same gearwheel pair, distinctive deviations in the characteristics can be investigated and related to single gearwheels (Albers et al. 2007a).

Tests have been conducted by using the micro test rig that has been developed within the Institute of Product Development Karlsruhe.

\section{Simulation}

The validation of the simulation results against the micro test rig has been performed through the radial composite

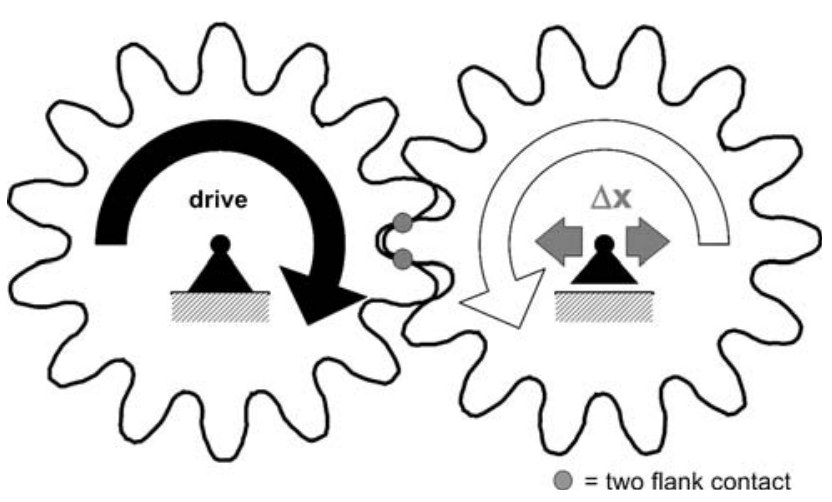

Fig. 5 Radial composite inspection 
inspection, as mentioned in the previous section. The runs have been simulated with slow rotational speed, so to keep the inertial forces as low as possible. From a numerical point of view, it is then possible to consider the test as a sequence of static load cases (Albers et al. 2007b). The pressing force acting on the flanks has been kept as low as possible, in order to avoid deformation on the profile of the teeth.

For this simulation, both a multi body simulation (MBS) with rigid bodies or an FE-Simulation are well suited. On the current work, and due to the "static" nature of the problem, the way over an FE-Simulation with the FEA Solver Abaqus has been chosen. The inertial effects are then taken out of consideration. It is also possible to divide the task on many parallel jobs within Abaqus, what reduces the wall-time needed for processing.

Because the model's complexity required in simulating a radial composite inspection is not previously known, and in hand of the geometrical data available, it has been decided to start with a simple model and run the simulations on a 2D basis. This makes also easier to model the joints of the system. On the computer modeling, the output gear remains fixed on a point and is free to rotate, connected to ground through a revolute joint. The input gear rotates with constant velocity, and is free to move on one radial direction. The pre-load or pressing force acts at the axis of this gear. Output measures of interest are the rotation angle on both gears and the linear displacement of the input gear.

A very fine FE-mesh on the contour of the gears allows a detailed representation of the measured, irregular profile of the teeth. Load paths and stress distribution are not of interest in this work, so the mesh on the inner parts of the gears is kept relatively coarse, in order to reduce the total amount of elements in the model. It could be also of benefit to consider both gears as rigid bodies, but the contact solver of choose is not capable to solve this particular problem with two rigid surfaces. Thus, only one gear and the spoke of the second one are considered rigid, while the teeth of the second gear are modeled with standard linearelastic material properties.

In order to assure the comparability of simulation and test results, the gears were coupled with the same orientation and tooth pairs, and then rotated one complete revolution. The center distance variations were measured, where zero means nominal center distance, and positive variation means longer distances.

Different tooth combinations on the gears were simulated and tested. Additionally, a series of simulations were conducted on selected tooth pairs, to gain an insight on the quality of the simulation process developed. Each of this series consisted on rotating the gears on both directions for one revolution, and extra runs with higher pressing forces.
The analysis of the simulation results for these parameters showed an exact concordance between the runs in the different directions, and pretty good agreement for the runs with altered pre-loads, as shown in Fig. 6. As expected, the center distance is slightly smaller for the cases with higher pressing forces.

As shown in Table 1, the number of increments needed by the solver to perform a $360^{\circ}$ rotation kept very stable for all the simulation cases. This can be used as an indication for the quality of the simulation process adopted as well as a sign that no extra difficult positions were encountered during the rotations.

The also very stable amount of required iterations shows that the convergence ratio of the model is not remarkably affected by the different teeth combinations.

The processes to measuring, modeling and simulating explained on the lines above were conducted both on the already described module 1 plastic gears, and on the ceramic gears of the CRC.

The smaller size of the CRC gears adds no additional effort to the simulation process, which behaves very stable in this case too. But the relative to their size bigger tolerances of the micro gears introduced new geometrical issues to the model. Cases have been found, where the contact between gears occurs on the outer sides of the teeth and not on the inner ones as expected, as shown in Fig. 7.

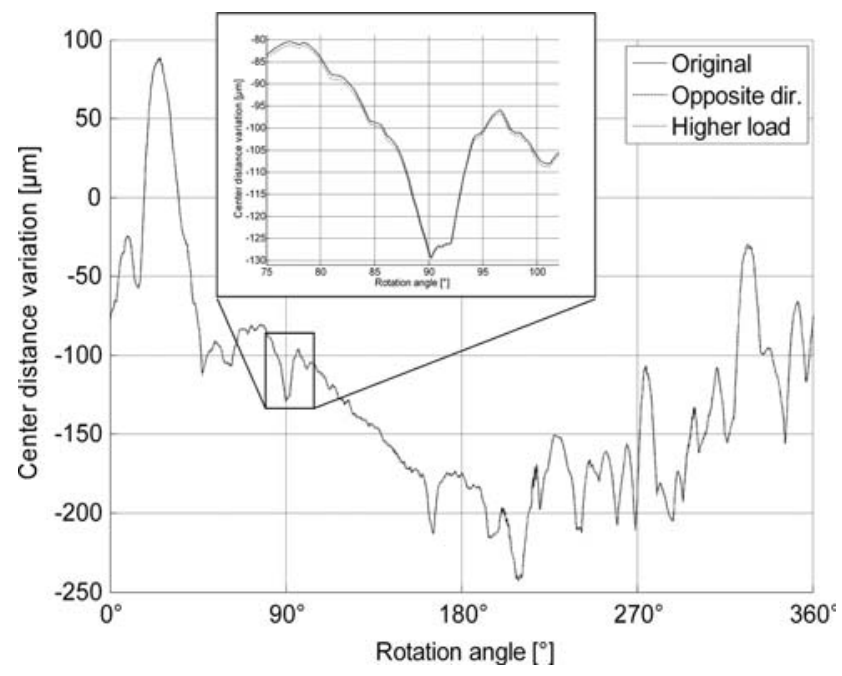

Fig. 6 Simulation series for the Module $1 \mathrm{~mm}$ gears

Table 1 Statistics for all the simulation series

\begin{tabular}{llrrrr}
\hline & \multicolumn{2}{l}{ Increments } & & \multicolumn{2}{l}{ Iterations } \\
\cline { 2 - 3 } \cline { 6 - 6 } \cline { 6 - 6 } & Mean & SD & & Mean & \multicolumn{1}{c}{ SD } \\
\hline Module $1.0 \mathrm{~mm}$ & 3220.0 & 27.726 & & 10012.3 & 120.821 \\
Module $0.169 \mathrm{~mm}$ & 3164.5 & 5.292 & & 9549.4 & 36.800 \\
\hline
\end{tabular}


Fig. 7 Undesirable engagement on the outer flanks due to profile deviation

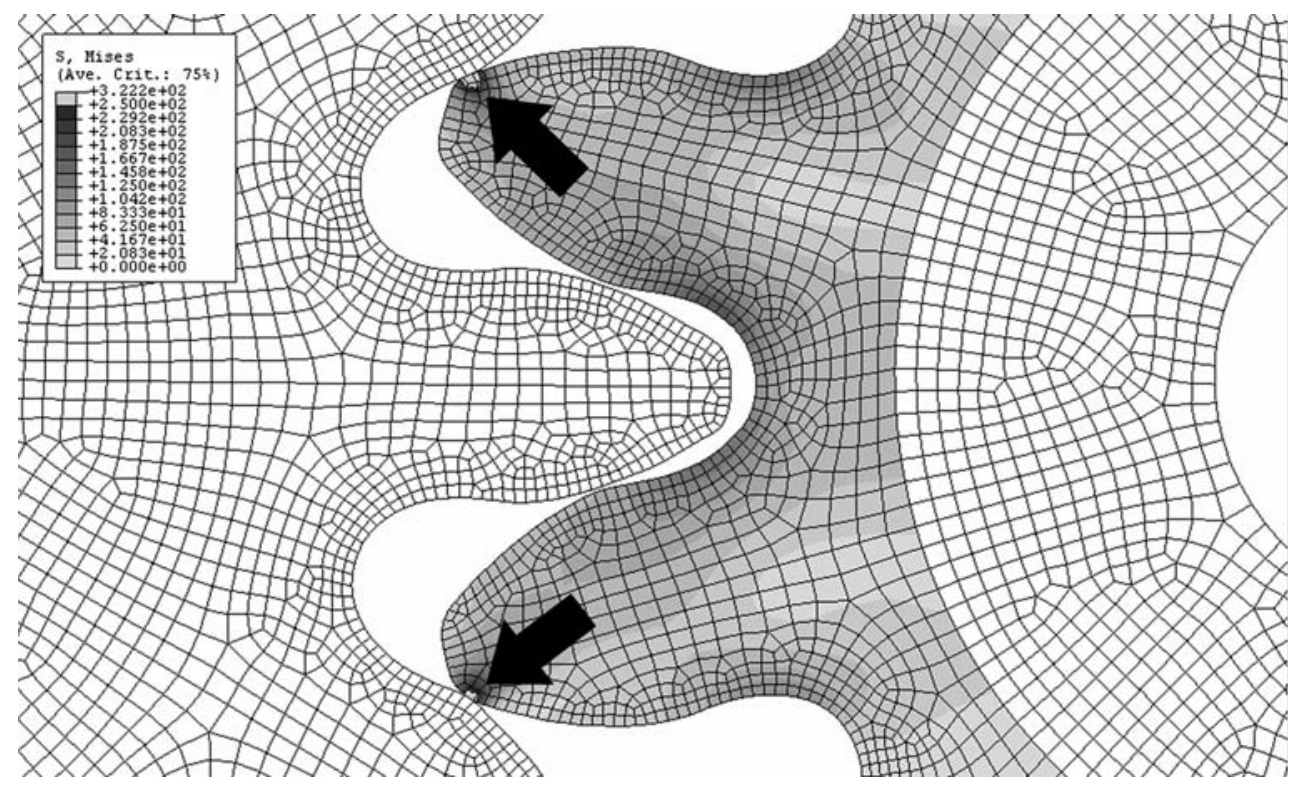

Fig. 8 Undesirable contact on the fillet zone due to profile deviation

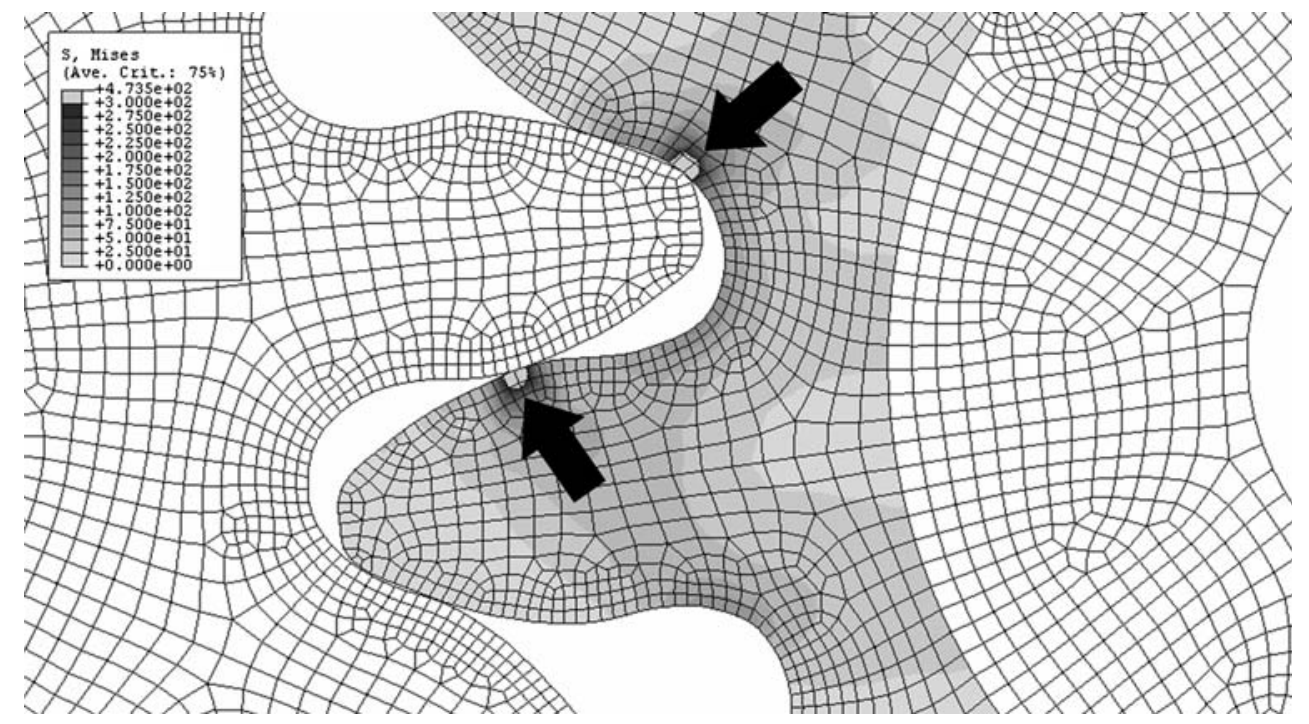

It is also possible, as depicted in Fig. 8, that the contact point lies wide under the base circle, in the middle of the fillet.

\section{Validation of simulation and test}

Based on the simulation results and the testing data, a comparison of the results for the center distance variation has been performed. For the plastic gears, this comparison shows good correlation between the simulated curves and the measured ones on the long wave domain. But for the much more interesting short wave domain, were the effects of the profile deviations are controlling, satisfying correlation could not be found. The compensation of the profile deviations along the faces of the teeth is believed to be one cause for this behavior. Due to the planar nature of the model, the contour variation along the width of the gear is not taken in account.

For the ceramic micro gears, significant deviations on both long wave and short wave domains have been found between the curves. See Fig. 9. For some sections of the curves, a good correlation even on the short wave domain could be found, as shown in Fig. 10. The causes for the significant long wave deviations are believed to lie on difficulties appeared on the gear mounting on the test rig. These induce then axes eccentricities and concentricity errors, which were not simulated. 


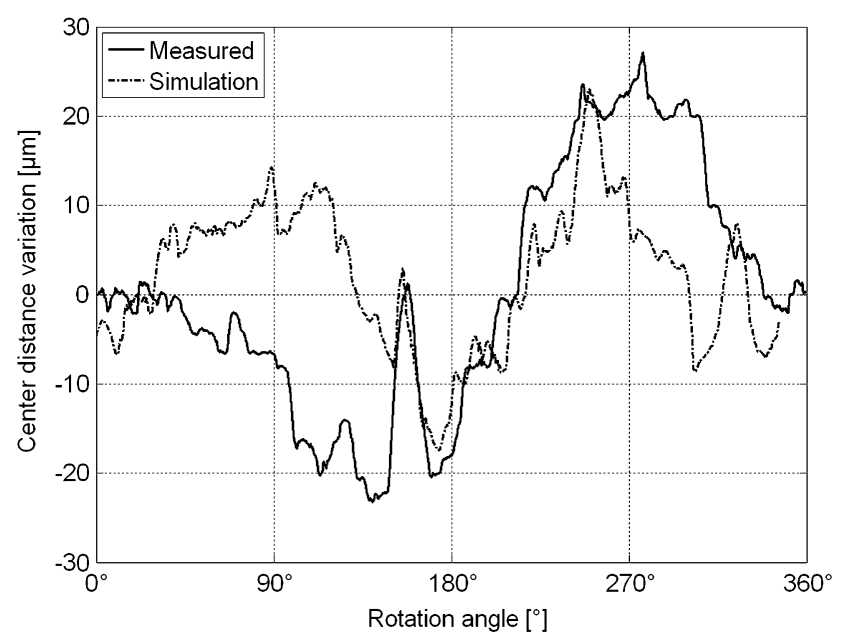

Fig. 9 Comparison between simulation and test for two micro gears based on a radial composite inspection

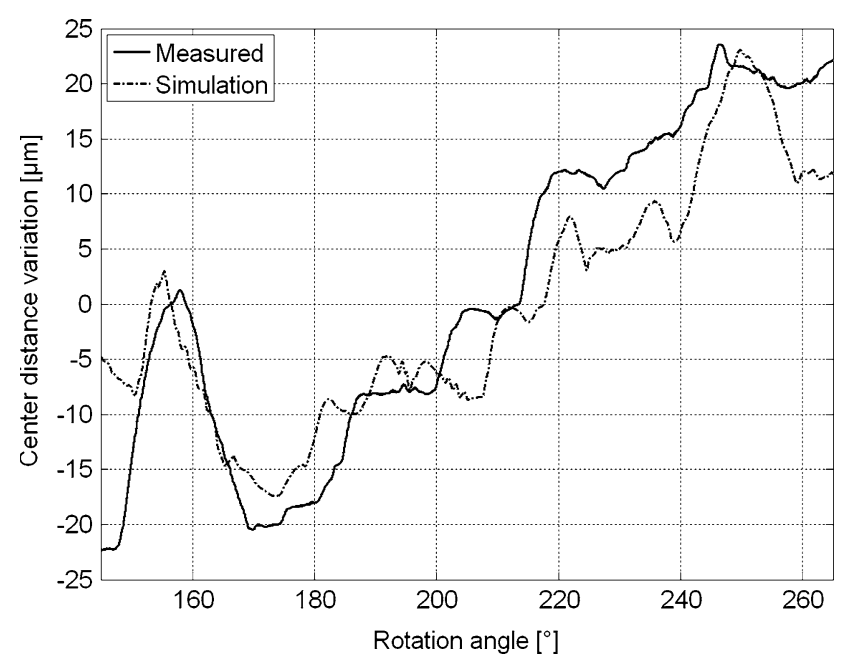

Fig. 10 Section with good correlation between simulated and measured results

This effect couldn't be detected earlier because of the relative big size of the plastic gears when compared to the magnitude of the mounting errors.

As in the tests with the plastic gears, the non complete correlation on the short wave domain could be related to the incomplete representation of the gear's geometry due to the two dimensional approximation.

\section{Conclusion}

The comparison of simulation and test of gear rolling indicates deviations within short and long wave domains. These can be ascribed to the reduction of the model from three to two dimensions. Deviations might also derive from inaccuracies when mounting micro gears. Further on, good correlation of test and simulation was demonstrated in some areas.

Because the two dimensional model appeared not being good enough to replicate the real gearing behavior, the following steps are aimed at modeling virtual gear wheels by superposition of several layers of contour measurements taken at different heights of the flank profile. Another alternative is to virtually engage a set of three-dimensionally modeled gear wheels, which will result in a much bigger computing effort.

The determined deviations excessing those of the gearing concerning eccentricity and runout have to be inspected by further examinations of the specimen connection, of the shaft mounting and of the shaft manufacturing. In this context the integration of such deviations into a simulative validation method has to be considered.

The integrative approach of validation of micromechanical systems in a simulative and experimental way is a first step towards the objective of a very early property assurance that enables a robust design. Based on this approach, also those influences that cannot be tested experimentally should be estimated through simulation in future. This could help to ensure the function fulfillment of micromechanical products.

Acknowledgments The authors would like to thank the German Research Foundation for supporting this work within the Collaborative Research Center "Development, Production and Quality Assurance of primary-shaped Micro Parts made of Metallic and Ceramic Materials" as well as all colleagues for providing specimens and measurement data.

\section{References}

Albers A, Burkardt N, Deigendesch T, Ellmer C, Hauser S (2007a) Validation of micromechanical systems, Int. Workshop on highaspect-ratio micro-structure technology HARMST, Besancon, France, 7-9 June 2007

Albers A, Enkler H-G, Leslabay P, (2007b) On the simulation of molded micro components and systems, Int. Workshop on highaspect-ratio micro-structure technology HARMST, Besancon, France, 7-9 June 2007

Marz J (2005) Mikrospezifischer produktentwicklungsprozess ( $\mu$ PEP) für werkzeuggebundene Mikrotechniken. Dissertation, IPEK Forschungsberichte Bd. 17, 2005

Mitutoyo Messgeräte GmbH (2007) Neuss, Germany. http://www. mitutoyo.de. Accessed 31 July 2007

Normenausschuss Feinmechanik und Optik (NaFuO) (1972a) Im DIN Deutsches Institut für Normung e.V. IN 58405: Stirnradgetriebe der Feinwerktechnik. Beuth Verlag, Berlin

Normenausschuss Feinmechanik und Optik (NaFuO) (1972b) Im DIN Deutsches Institut für Normung e.V. DIN 58420: Lehrzahnräder zum Prüfen von Stirnrädern der Feinwerktechnik. Beuth Verlag, Berlin

Normenausschuss Antriebstechnik (NAN) (1987) Im DIN Deutsches Institut für Normung e.V. DIN 3960: Begriffe und Bestimmungsgrößen für Stirnräder (Zylinderräder) und Stirnradpaare 
(Zylinderradpaare) mit Evolventenverzahnung. Beuth Verlag, Berlin

Schoenberg IJ (1969) Approximations with special emphasis on spline functions, proceedings of a symposium conducted by the mathematics research center, United States Army, Madison, 5-7 May 1969

VDI (2001) VDI/VDE-Gesellschaft Mess- und Automatisierungstechnik (GMA) VDI/VDE 2608: Einflanken- und Zweiflanken-
Wälzprüfung an Zylinderrädern. Kegelrädern, Schnecken und Schneckenrädern/Tangential composite and radial composite inspection of cylindrical gears, bevel gears, worms and worm wheels. Beuth Verlag, Berlin

Carl Zeiss AG (2007) Oberkochen, Germany. http://www.zeiss.de. Accessed 31 July 2007 